\title{
Razonamiento Configural y Espacio de Trabajo Geométrico en la Resolución de Problemas de Probar
}

\author{
Configural Reasoning and Geometric Workspace \\ in the Proof's Problem Resolution
}

\author{
Juan Prior Martínez* \\ ORCID iD 0000-0001-8734-6407 \\ Germán Torregrosa Gironés** \\ ORCID iD 0000-0002-2509-2031
}

\begin{abstract}
Resumen
La transición desde las primeras justificaciones de propiedades geométricas en el entorno escolar hacia la demostración matemática en un contexto deductivo es un problema profusamente estudiado. A partir de la Teoría de los Paradigmas y Espacio de Trabajo Geométrico, que nos proporciona un marco teórico atendiendo al ambiente institucional en que se desarrolla la actividad geométrica, utilizamos el modelo de Razonamiento Configural para estudiar el espacio de trabajo geométrico personal del resolutor de una tarea de probar una propiedad geométrica. Describimos la organización discursiva de las respuestas de estudiantes de secundaria a un cuestionario de cuatro problemas, en los que se pide probar una propiedad geométrica, y determinamos los razonamientos configurales que realizan para obtener dichas respuestas. Este análisis nos permite aportar evidencias acerca del tránsito que deben recorrer los estudiantes desde las primeras justificaciones experimentales en la Geometría Natural hasta el razonamiento matemático válido propio de la Geometría Axiomática Natural. El Razonamiento Configural se muestra como un modelo teórico con una gran capacidad para abordar la articulación entre visualización y razonamiento.
\end{abstract}

Palabras clave: Razonamiento Configural. Didáctica de la Geometría. Prueba Matemática. Espacio de Trabajo Geométrico.

\begin{abstract}
The transition from the first justifications of geometric properties in the school environment to the mathematic proof in a deductive context is an issue widely studied. From the Theory of Paradigms and Geometric Workspace, which provides a framework regarding the institutional environment in which the geometric activity is developed, we use the model of Configural Reasoning to study the resolver's geometric workspace when facing a task of proving a geometric property. We describe the discourse organization of high school students' responses to a four-task questionnaire in which they were asked to prove a geometric property, and we determine the configural reasoning that led them to those responses. This analysis allows us to provide evidence on the transition that students must undergo from their first experimental justifications in Natural Geometry to the valid

\footnotetext{
* Diploma de Estudios Avanzados por la Universidad de Alicante (UA), Alicante. Profesor Asociado de la Facultad de Educación de la Universidad de Murcia (UMU), Murcia, España. Dirección postal: Facultad de Educación, Universidad de Murcia, Calle Campus Universitario, 12, Murcia, España, código postal 30100. Email: juan.prior@um.es.

*** Doctor en Matemáticas por la Universidad de Alicante (UA). Catedrático de Escuela Universitaria de la Universidad de Alicante (UA), Alicante, España. Dirección postal: Facultad de Educación, Universidad de Alicante, Calle Aeroplano, s/n, San Vicente del Raspeig, Alicante, código postal 03690. E-mail: german.torregrosa@ua.es.
} 
mathematical reasoning proper of Natural Axiomatic Geometry. The Configural Reasoning is shown as a theoretical model with a great capacity for addressing the articulation between visualization and reasoning.

Keywords: Configural Reasoning. Geometry Didactics. Mathematical Proof. Geometric Workspace.

\section{Introducción}

La literatura en torno al razonamiento en contexto geométrico es muy extensa; la mayoría de investigaciones sobre la enseñanza y el aprendizaje de la demostración se han realizado en el dominio de la geometría euclidiana (LARIOS, 2005; MARIOTTI; PEDEMONTE, 2019; STYLIANIDES; STYLIANIDES, 2009; MARRADES; GUTIÉRREZ, 2000; HERSHKOWITZ; PARZYSZ; VAN DORMOLEN, 1996; PARASKEVI; GAGATSIS, 2014; HOUDEMENT; KUZNIAK, 1999, 2006; DUVAL, 1998, 2006, 2007).

Todas ellas ponen de manifiesto la complejidad de la transición que el estudiante de geometría ha de realizar, desde las primeras aproximaciones al trabajo geométrico en un contexto real con objetos reales y procedimientos de verificación asociados a la intuición o al uso de instrumentos de medida, hasta la geometría formal donde los objetos matemáticos tienen una entidad, independiente de su existencia real o no, y los procedimientos de validación son los propios de la deducción formal en un conjunto de axiomas completo.

La Geometría involucra tres clases de procesos cognitivos: procesos de visualización, de razonamiento y de construcción (DUVAL, 1998). En trabajos anteriores, Torregrosa y Quesada (2007) y Torregrosa, Quesada y Penalva (2010) se ha propuesto un modelo que explica la coordinación de los procesos de visualización involucrados en la resolución de problemas de prueba de propiedades geométricas a partir de respuestas de estudiantes para maestro de Educación Primaria. Esta coordinación desemboca en un proceso de razonamiento que denominamos razonamiento configural. Posteriormente, este modelo se ha extendido a la resolución de problemas empíricos (SAORÍN et al., 2017), mostrando un gran potencial explicativo para describir el razonamiento en cualquier tipo de actividad geométrica. Tratando de poner a prueba el modelo teórico con estudiantes de últimos cursos de educación secundaria obligatoria (15-16 años), Prior y Torregrosa (2013) refinaron la clasificación de los diferentes desenlaces en que puede desembocar el razonamiento configural. El estudio de las respuestas de alumnos de una etapa distinta mostró que el razonamiento configural se ve influenciado por los procedimientos de validación que se aceptan en el contexto matemático de la actividad, así como por la organización discursiva que demanda la tarea, bien sea esta una comprobación, una explicación o una prueba matemática. Estas influencias produjeron 
una amplia diversidad de respuestas en alumnos de la misma edad y que atendían a las mismas clases. Esta situación nos ha llevado a revisar nuestro modelo de razonamiento configural añadiendo una nueva variable: el espacio de trabajo geométrico (ETG), noción introducida por Houdement y Kuzniak $(2003$, 2006) con el objetivo de comprender lo que, desde un punto de vista didáctico, se pone en juego alrededor del trabajo matemático en un marco escolar.

El objetivo de este artículo es analizar el razonamiento configural que desarrollan alumnos enfrentados a tareas de justificación de propiedades geométricas para describir características de los espacios de trabajo personales en los que producen sus respuestas. Para ello, realizaremos un análisis de las respuestas de los estudiantes y describiremos los ciclos coordinados de aprehensiones operativas y discursivas que conforman sus razonamientos configurales con la finalidad de identificar características del contexto matemático (ETG) en que se produce la respuesta de los alumnos.

Los resultados de este análisis nos ayudarán a entender la diversidad de respuestas de los estudiantes cuando resuelven problemas de geometría, y a identificar los obstáculos epistemológicos que deben superar desde las primeras verificaciones experimentales hasta la demostración matemática.

\section{Marco Teórico}

El estudio de la coordinación necesaria entre procesos de visualización para la resolución de problemas de prueba de propiedades geométricas en un contexto de geometría deductiva ha permitido desarrollar un modelo teórico al que se ha denominado razonamiento configural (TORREGROSA; QUESADA, 2007; TORREGROSA; QUESADA; PENALVA, 2010). Tal proceso implica la coordinación de aprehensiones discursivas y operativas (DUVAL, 1998; PRIOR; TORREGROSA, 2013), y puede desembocar en distintos desenlaces:

1. La coordinación da una solución al problema. Y, en este caso, se distinguen:

a. Truncamiento, que se produce cuando la coordinación proporciona la idea para resolver deductivamente el problema.

b. Conjetura sin demostración, cuando la coordinación permite resolver el problema aceptando conjeturas mediante percepción simple.

2. La coordinación no consigue ninguna solución. Denominamos a esta situación bucle. 
El desenlace Conjetura sin demostración se puede dividir en dos subcategorías: conceptual y empírica, dependiendo de si la coordinación está o no inmersa en un procedimiento discursivo de carácter deductivo, en el primer caso, y de la utilización o no de procedimientos de verificación experimentales, en el segundo (PRIOR; TORREGROSA, 2013).

Este modelo ha sido testado en diferentes investigaciones, analizando respuestas de alumnos a problemas de demostración de propiedades geométricas (LLINARES; CLEMENTE, 2014; CLEMENTE; TORREGROSA; LLINARES, 2015) y se ha extendido a la resolución de problemas empíricos (TORREGROSA, 2017; SAORÍN; TORREGROSA; QUESADA, 2017) permitiendo identificar algunas características de cómo funciona la relación entre las figuras, los hechos geométricos y la generación de relaciones lógicas entre ellos (CLEMENTE; LLINARES, 2015).

Desde una perspectiva epistemológica, cualquier actividad geométrica tiene lugar en un contexto determinado. En el caso particular de la demostración de propiedades geométricas, algunos autores se centran en el aspecto de constructo social de la justificación, de manera que lo que constituye una demostración depende del contexto matemático y cultural en el que uno está trabajando (ZAZKIS; WEBER; MEJÍA-RAMOS, 2016); el aprendizaje de la demostración se ve como una práctica sociocultural que se lleva a cabo y se condiciona por la comunidad en la que se inscribe (FIALLO; CAMARGO; GUTIÉRREZ, 2013).

Otros, Harel y Sowder (1998), se centran en asuntos cognitivos referidos a los esquemas de demostración. Definen esquema de demostración como lo que permite asegurarse, en el sentido de eliminar sus dudas acerca de la veracidad de una afirmación matemática, y persuadir, eliminar las dudas de otros sobre dicha afirmación. Este concepto les permite extraer un conjunto de categorías de demostraciones empíricas y deductivas.

Un aspecto interesante en la definición de esquema de demostración es la consideración explícita de una comunidad como unidad de elaboración de demostraciones, ya que para ello es necesario que los individuos que la integran se pongan de acuerdo en aspectos cruciales como qué es demostrar, cómo se demuestra una afirmación matemática y qué características debe poseer un argumento para ser reconocido como una demostración válida.

Desde este punto de vista, la actividad de producir una demostración es una práctica cuyas características dependen del ámbito institucional en donde se lleve a cabo. En torno a la idea de institución en la que se desarrolla la actividad matemática, Houdement y Kuzniak (1999, 2006) construyen el concepto de espacio de trabajo geométrico (ETG) con el que 
pretenden comprender lo que, desde el punto de vista didáctico, se pone en juego alrededor del trabajo matemático en un contexto geométrico escolar.

Aunque el término Geometría está presente en todos los currículos matemáticos desde la etapa infantil hasta la enseñanza secundaria, e incluso en determinados programas universitarios, obviamente no tiene el mismo significado en todos ellos. En sus investigaciones, describen tres paradigmas geométricos, articulando esta división en relación a diferencias filosóficas, en relación con las creencias de la comunidad matemática, epistemológicas, con respecto a los contenidos matemáticos, y cognitivas, que conciernen al pensamiento del sujeto que resuelve tareas geométricas.

Estos paradigmas determinan un espacio de trabajo geométrico de referencia, entendido como un ambiente organizado para permitir el trabajo de las personas que resuelven problemas geométricos (KUZNIAK, 2008). Así pues, tenemos tres tipos de geometrías o paradigmas geométricos, provisto cada uno de un ETG que describimos a continuación.

- Geometría Natural o Geometría I. Aquí los procedimientos de validación son de naturaleza perceptiva y están, por tanto, íntimamente relacionados con la realidad, con o sin el uso de instrumentos. Es un sistema no axiomático donde los objetos tienen una naturaleza material.

- Geometría Axiomática Natural o Geometría II. Los procedimientos de validación se basan en las leyes hipotético-deductivas en un sistema axiomático. El sistema axiomático está tan conectado a la realidad como sea posible, y, probablemente, sea un sistema incompleto. Los objetos geométricos son ideales, sujetos, por tanto, no a la experiencia real sino a la definición.

- Geometría Axiomática Formal o Geometría III. Por supuesto, los objetos en este paradigma son también ideales y la única validación es el razonamiento hipotéticodeductivo. Aquí, cualquier tipo de conexión con la realidad se vuelve secundario. El conjunto de axiomas es completo y sin relación con nuestros sentidos, lo que diferencia este paradigma de la Geometría Axiomática Natural. La característica central de la validación en este contexto es la consistencia, es decir, la ausencia de contradicciones.

La Geometría que una institución (escolar o profesional) tiene en mente está descrita en los ETG de referencia: GI, GII y GIII, que representan el espacio de trabajo definido de manera ideal en función de criterios matemáticos. Estos ETG de referencia deben ser acondicionados en un ETG idóneo en las clases, definido en términos didácticos, donde cada alumno trabaja en su ETG personal. 
La Geometría Axiomática Formal o GIII es el ETG de referencia característico de la Geometría en etapas universitarias, especialmente con el estudio de Geometrías no euclídeas, por lo que la atención en las matemáticas escolares se centra en los dos primeros: Geometría I y II (KUZNIAK, 2008), así como en las dificultades que enfrentan los alumnos en el tránsito desde la Geometría Natural, propia de la etapa Primaria, hasta la Geometría Axiomática Natural, que se pretende alcanzar durante la etapa Secundaria. Es decir, se trata de abordar la aparición de la deducción, que permite la transición desde el ver al conocer (PARZYSZ, 1988).

Con respecto a la noción de prueba matemática, en nuestro trabajo adoptamos la descripción de Duval (2007) acerca de lo que es un razonamiento matemático válido. Conviene, por tanto, desarrollar lo que se entiende por leyes hipotético-deductivas en un sistema axiomático, ya que estas son el procedimiento de validación de proposiciones característico del ETG denominado Geometría Axiomática Natural y éste es el ETG de referencia en la enseñanza secundaria.

Hay diferentes modos de pasar de una o varias proposiciones a otra, pero no todas son permitidas en la construcción de una verdadera prueba matemática. Cualquier razonamiento deductivo implica dos niveles bien distintos de organización discursiva: el nivel de organización de varias proposiciones en un paso de deducción y el nivel de organización de varios pasos en una prueba. Para la clasificación de las respuestas de los estudiantes se han utilizado las siguientes categorías, que se apoyan en los trabajos de Harel y Sowder (1998) y Balacheff (1988):

- Comprobación. Verificación empírica, mediante el uso de instrumentos, de la afirmación matemática para el caso particular del enunciado propuesto. Propia de la Geometría Natural.

- Explicación. Discurso no deductivo (no sujeto a la organización discursiva propia de la prueba matemática) cuya finalidad es convencer de la veracidad de la proposición en base a argumentos visuales.

- Prueba Ingenua. Aunque posee la estructura multinivel de la demostración matemática, la validación perceptiva de algunas proposiciones nos impide dar categoría de prueba matemática a este tipo de discurso.

- Demostración. Discurso caracterizado por la estructura multinivel específica de la demostración matemática. Propia de la Geometría Axiomática Natural.

En este estudio pretendemos mostrar que el razonamiento configural que desarrolla un alumno enfrentado a la resolución de una tarea de probar se relaciona con las características 
del espacio de trabajo en el que éste considera implícitamente que la tarea tiene lugar. Si bien la división de la actividad geométrica en tres espacios de trabajo geométrico se ajusta a la experiencia educativa, la observación del trabajo de los alumnos permite afirmar que, en la práctica, esta división no es inflexible, siendo fácil observar tareas y respuestas en las que cohabitan características de distintos ETG. El análisis de las respuestas de los alumnos nos ha permitido describir las características del razonamiento configural propio de la Geometría Natural, del característico de la Geometría Axiomática Natural y, de suma importancia, de momentos de tránsito entre estos dos espacios de trabajo geométrico.

\section{Método}

La investigación se enmarca dentro de la agenda de investigación centrada en la caracterización de los procesos cognitivos que ponen de manifiesto los estudiantes cuando resuelven problemas de geometría (HERSHKOWITZ; PARZYSZ; VAN DORMOLEN, 1996; DUVAL, 1998, 2007; PARASKEVI; GAGATSIS, 2014; CLEMENTE; TORREGROSA; LLINARES, 2017). Esta aproximación pone de relieve la importancia dada a la relación entre lo visual y lo analítico en el estudio de los procesos de resolución de problemas de geometría. Desde este punto de vista, se asume que la coordinación entre lo visual y lo analítico puede ser relevante para el desarrollo de las pruebas matemáticas.

\subsection{Participantes y contexto}

En el estudio participaron 38 alumnos de $3^{\circ}$ y $4^{\circ}$ de Educación Secundaria Obligatoria, con edades comprendidas entre los quince y dieciséis años. Estos estudiantes no han recibido instrucción directa sobre la demostración matemática en general, ni sobre la resolución de problemas de probar en contexto geométrico en particular. Los participantes resolvieron un cuestionario propuesto por el profesor-investigador en una sesión de la clase de matemáticas.

\subsection{Instrumento}

Los estudiantes resolvieron un cuestionario con cuatro problemas en los que se demandaba que probasen una propiedad geométrica. Cada uno de los enunciados iba acompañado de una figura. Los problemas se seleccionaron de manera que los alumnos disponían de los conocimientos geométricos necesarios para su resolución. Después de la 
realización del cuestionario, el investigador entrevistó a los alumnos acerca del procedimiento de validación utilizado en cada una de las afirmaciones de sus respuestas (Figura 1).

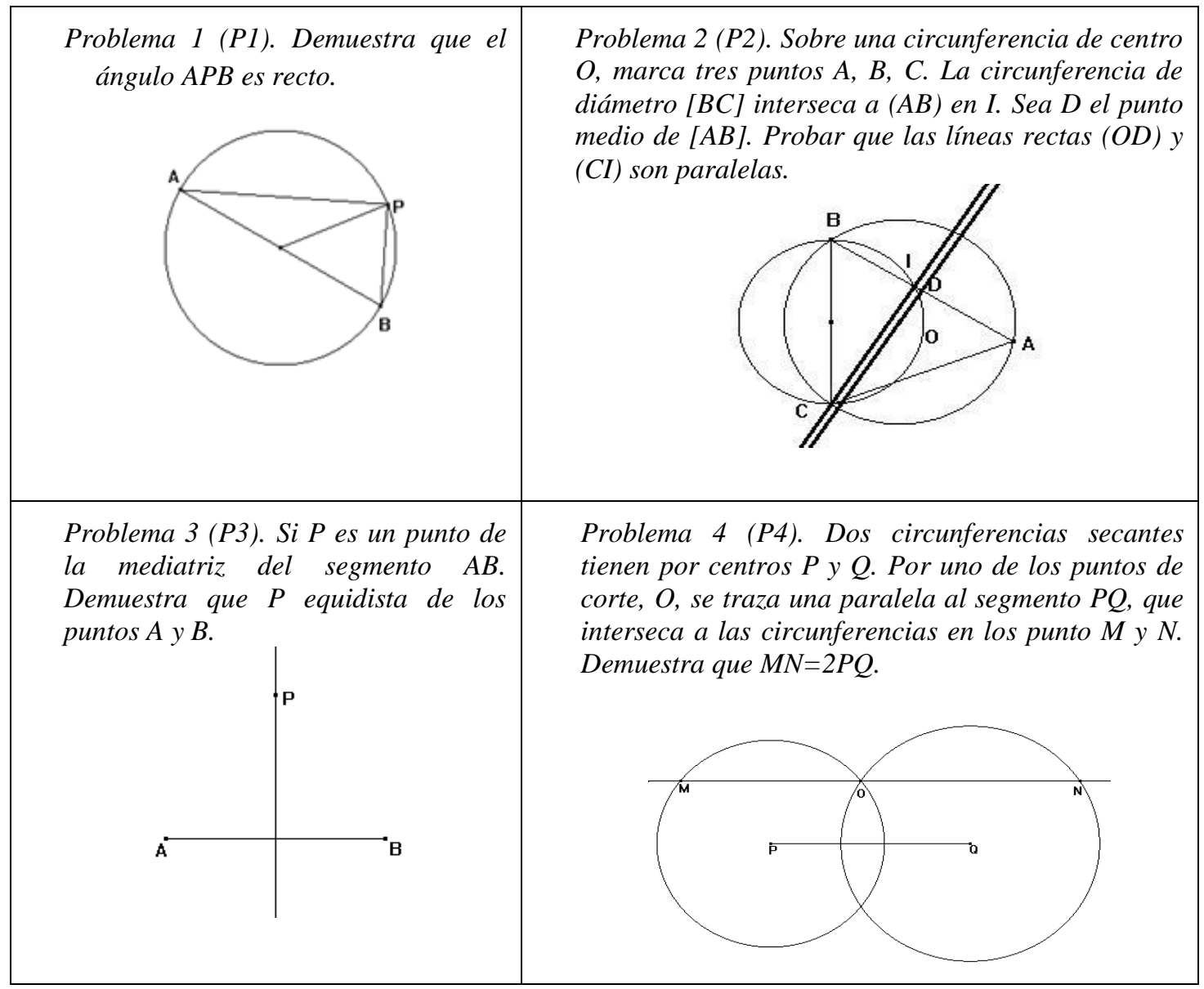

Figura 1 - Relación de problemas del cuestionario

Fuente: Datos de la investigación

En la selección de los problemas se tuvo en cuenta que la tesis a demostrar fuese visualmente evidente (P2 y P3) y no evidente (P1 y P4) y que hubiese necesidad de modificar la configuración inicial, añadiendo nuevos elementos para su resolución (P3 y P4) o no (P1 y P2).

\section{Análisis}

Para ilustrar las fases del análisis usamos como ejemplo la producción de uno de los estudiantes. El análisis se ha realizado en cuatro fases. En primer lugar, se ha realizado una transcripción y segmentación de las respuestas escritas dadas por los estudiantes con una doble finalidad: por un lado, identificar los ciclos coordinados de aprehensiones operativas y discursivas que conforman el razonamiento configural seguido por los estudiantes (TORREGROSA; QUESADA; PENALVA, 2010); por otro, identificar las diferentes 
afirmaciones matemáticas de la respuesta, con el fin de establecer el procedimiento de validación usado (Figura 2).

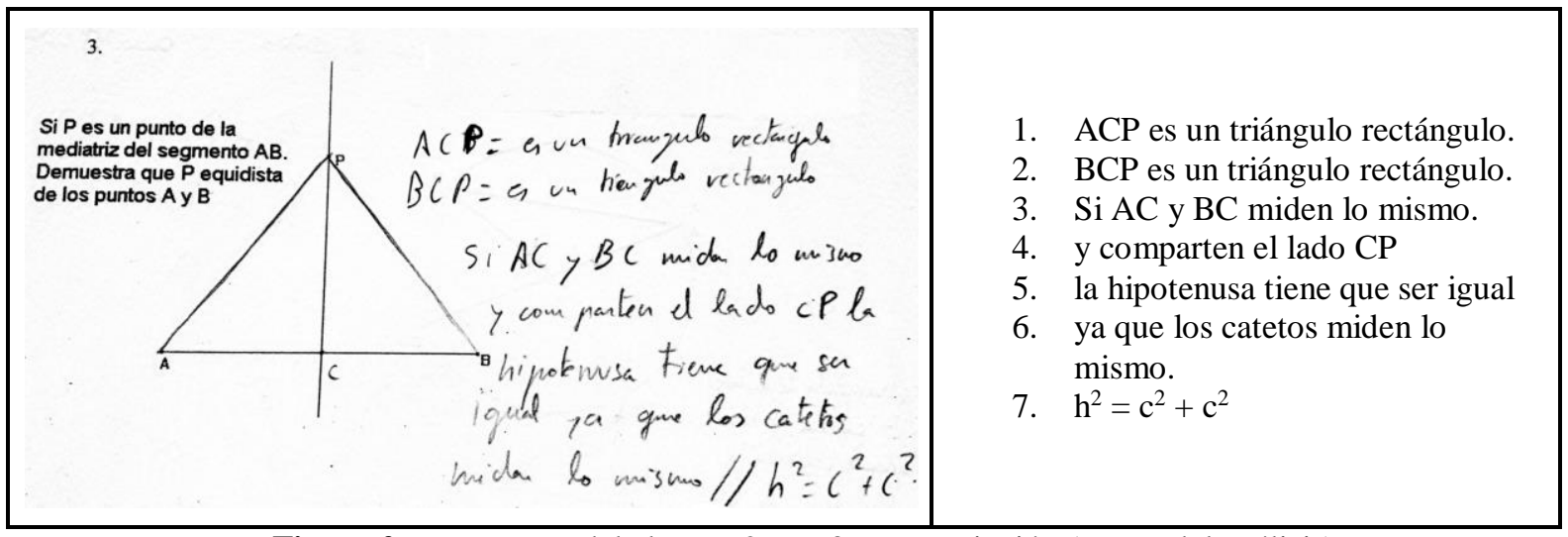

Figura 2 - Respuesta del alumno 26 a P3 y transcripción (Fase I del análisis)

Fuente: Datos de la investigación

En la segunda fase se ha entrevistado a cada uno de los estudiantes con el fin de establecer el procedimiento de validación que ha utilizado para cada una de las afirmaciones de su respuesta. En el caso de la respuesta del alumno 26, a P3 se pudo establecer que todas las afirmaciones que se realizaban se basaban en el procedimiento de validación que denominamos deductivo (Cuadro 1).

\begin{tabular}{|l|l|}
\hline Afirmación matemática & Procedimiento de validación \\
\hline ACP es un triángulo rectángulo & $\begin{array}{l}\text { En la entrevista manifiesta que el ángulo ACP es } \\
\text { recto "ya que la mediatriz es perpendicular al } \\
\text { segmento". Inferimos que usa el procedimiento de } \\
\text { validación deductivo }\end{array}$ \\
\hline Si AC y BC miden lo mismo & $\begin{array}{l}\text { Afirma que "la mediatriz siempre corta al segmento } \\
\text { en la mitad". } \\
\text { Procedimiento de validación deductivo }\end{array}$ \\
\hline
\end{tabular}

Cuadro 1 - Fragmento del análisis de los procedimientos de validación en la respuesta a P3 del alumno 26 (Fase II del análisis)

Fuente: Datos de la investigación

En la tercera fase realizamos un análisis de los ciclos de aprehensión operativa/discursiva del razonamiento configural. En primer lugar, realiza una aprehensión operativa de cambio figural al añadir los segmentos AP y BP a la figura, así como el punto C (Figura 3).

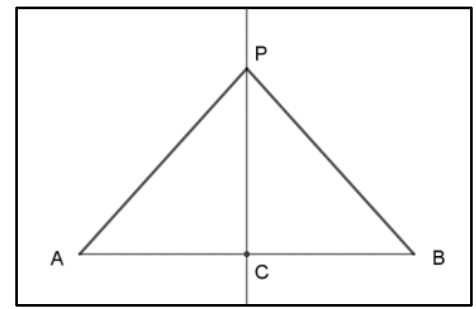

Figura 3 - Aprehensión operativa de cambio figural

Fuente: Datos de la investigación 
A continuación, realiza una aprehensión operativa de identificación al separar las subconfiguraciones formadas por los triángulos APC y BCP (Figura 4).

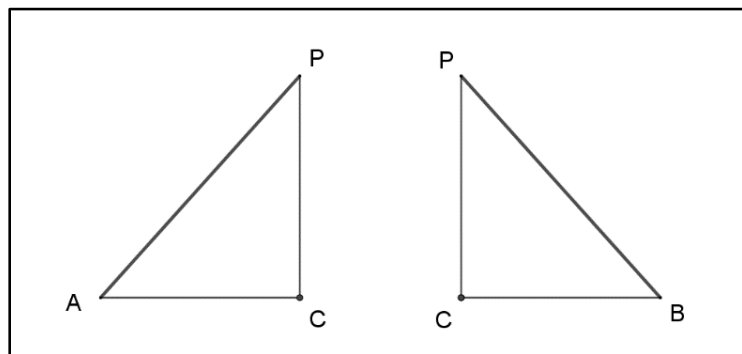

Figura 4 - Aprehensión operativa de identificación Fuente: Datos de la investigación

Seguidamente, realiza aprehensiones discursivas indicando que dichas subconfiguraciones son triángulos rectángulos (Figura 5).

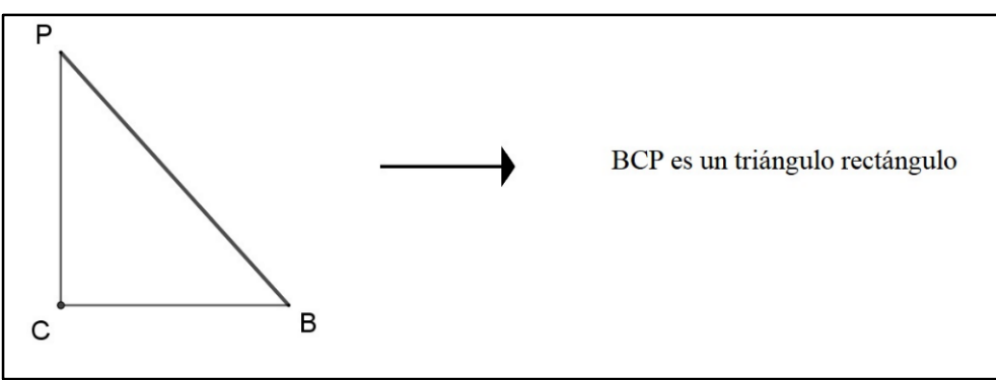

Figura 5 - Aprehensión discursiva con cambio de anclaje de visual a discursivo Fuente: Datos de la investigación

Por último, realiza una aprehensión discursiva asociando el teorema de Pitágoras a ambos triángulos (Figura 6).

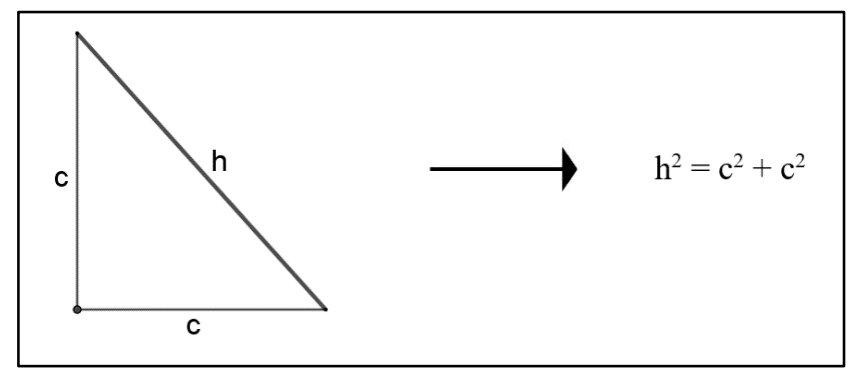

Figura 6 - Aprehensión discursiva con cambio de anclaje de visual a discursivo Fuente: Datos de la investigación

El razonamiento configural de este estudiante en respuesta a la tarea desemboca en un truncamiento que le permite resolver deductivamente el problema. La coordinación de ciclos de aprehensiones operativas y discursivas le ha llevado a deducir que los dos triángulos ACP y BCP son triángulos rectángulos con catetos iguales, aunque comete un error al asignar la misma incógnita, c, a los dos catetos, que no son necesariamente congruentes. En esta situación, el teorema de Pitágoras le garantiza la congruencia de las hipotenusas, que es la 
tesis que pretende demostrar. En la última fase, realizamos el análisis de la organización discursiva de su respuesta:

Paso 1: Premisa: ACP es un triángulo rectángulo $\rightarrow$ Teorema de Pitágoras $\rightarrow$ Conclusión: $A P^{2}=A C^{2}+C P^{2}$

Paso 2: $B C P$ es un triángulo rectángulo $\rightarrow$ Teorema de Pitágoras $\rightarrow B P^{2}=B C^{2}+C P^{2}$

Las conclusiones de los pasos 1 y 2 se convierten en premisas del Paso 3 donde, utilizando que $A C=B C$ (definición de mediatriz), podemos concluir que $A P=B P$ que es la tesis que se pretende demostrar (Análisis de la organización discursiva).

Si bien la falta de rigor en la redacción es la habitual en la mayoría de alumnos de estas edades, podemos observar en el discurso las características propias de la prueba matemática: nivel local (paso deductivo), donde cada afirmación adquiere una de las tres categorías (premisa, definición/teorema o conclusión) y nivel global (organización de varios pasos), donde las conclusiones de pasos anteriores son reutilizadas como premisas en pasos siguientes.

\section{Resultados}

A continuación, presentamos los resultados del análisis realizado en función de los desenlaces del razonamiento configural identificados. El contexto en que se enmarca una tarea (ETG idóneo) impone la validez de unos procedimientos de validación frente a otros, y esto implica la pertinencia de unos razonamientos configurales y no otros, por lo que hemos podido inferir algunas características del ETG personal en el que se produce la respuesta a la tarea a partir del razonamiento configural realizado.

Un tipo de razonamiento configural (Truncamiento Naif, CsDEmpírica, CsDConceptual o Truncamiento) produce un tipo de discurso específico (Comprobación, Explicación, Prueba Ingenua o Demostración). Además, el tipo de respuesta que demanda la tarea, dependiente del espacio de trabajo geométrico en el que es planteada, determina el tipo de razonamiento configural necesario. De este modo, nuestro modelo se convierte en un instrumento que nos podría permitir, a partir de las respuestas, inferir el razonamiento que el resolutor desarrolla, su comprensión de la demanda de la tarea y, por tanto, determinar el estadio en que se encuentra el estudiante en relación con los espacios de trabajo geométrico.

A continuación, mostramos las características de los binomios encontrados, analizando algunas respuestas que ilustran esta relación entre los desenlaces del razonamiento configural y las distintas organizaciones discursivas: 
Truncamiento Naif $\leftrightarrow$ Comprobación: este binomio se caracteriza por un razonamiento configural que desemboca en un truncamiento naif. Éste consiste en la realización de una aprehensión operativa para identificar la subconfiguración relacionada con la tesis a verificar, seguida de su validación experimental para el caso particular proporcionado por el enunciado. El discurso que el estudiante genera, propio de la Geometría Natural, no es más que la conversión al lenguaje natural del razonamiento configural realizado. Véase la Figura 7.

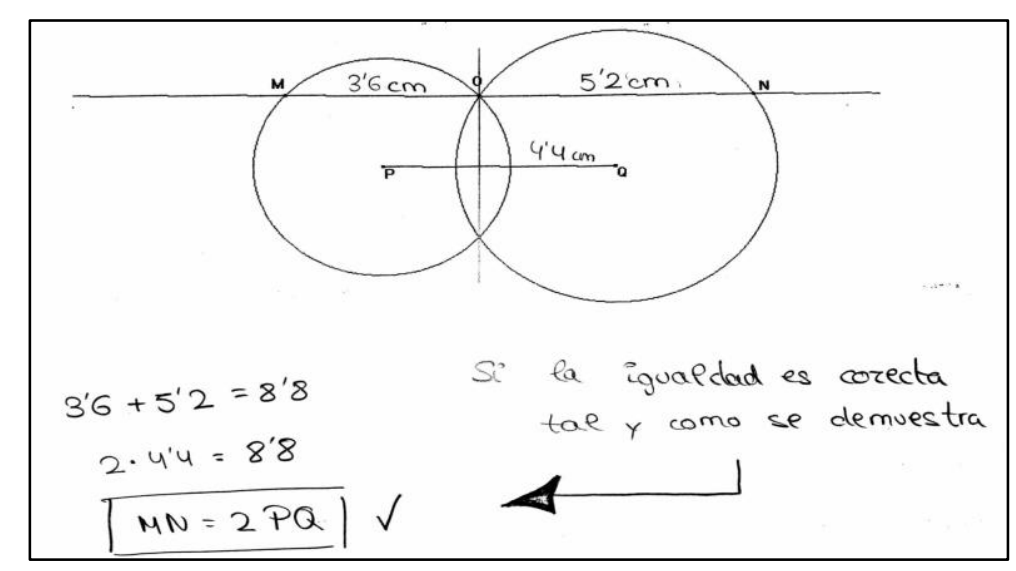

Figura 7 - Respuesta del alumno 6 a P4

Fuente: Datos de la investigación

Su razonamiento configural consiste en la realización de la aprehensión operativa de identificación que selecciona los dos elementos geométricos: segmentos MN y PQ a los que se refiere la proposición a demostrar. Tras esto, se limita a medir su longitud, usando un instrumento de medida (regla graduada). Su discurso consiste en la traducción de las acciones que realiza sobre la figura. Obtiene una medida de $8,8 \mathrm{~cm}$ para el segmento $\mathrm{MN}$, a partir de los segmentos que lo conforman $(\mathrm{MO}=3,6 \mathrm{~cm}$ y $\mathrm{ON}=5,2 \mathrm{~cm})$ y de $4,4 \mathrm{~cm}$ para el segmento PQ. Por tanto, concluye que la tesis del enunciado es cierta puesto que $8,8=2 \cdot 4,4$. Esto supone una muestra de que el estudiante asume las características de la Geometría Natural, utilizando la imagen que acompaña al enunciado como objeto de estudio y validación.

Esta situación muestra un problema, ya señalado, de la enseñanza de la geometría: la diferencia entre la tarea que propone el profesor y lo que el alumno entiende que debe realizar. Profesor y alumno trabajan en espacios de trabajo geométrico diferentes. En este caso, la palabra Demuestra en lugar de Comprueba, y las condiciones iniciales del problema: dos circunferencias secantes...; Se traza una paralela al segmento $P Q$ que interseca... son indicadores que nos muestran que este problema se plantea en el ETG Axiomático Natural. Sin embargo, el hecho de que el alumno no haga ninguna mención a las hipótesis en su respuesta nos indica que su ETG personal se enmarca en la Geometría Natural. 
Conjetura sin Demostración Empírica $\leftrightarrow$ Explicación: se produce un razonamiento configural en el que no es necesario un conocimiento geométrico explícito (definición, teorema,...), lo que se traduce en un discurso en el que no se observan los niveles propios de la prueba matemática. El discurso es una explicación, que puede resultar más o menos convincente en función de la evidencia de los argumentos visuales, pero no es una demostración desde el punto de vista de la organización discursiva. En el discurso podemos observar el nivel global de la estructura discursiva de la prueba matemática, pero no se observa el nivel local o paso deductivo.

En otra respuesta al mismo problema (Figura 8):

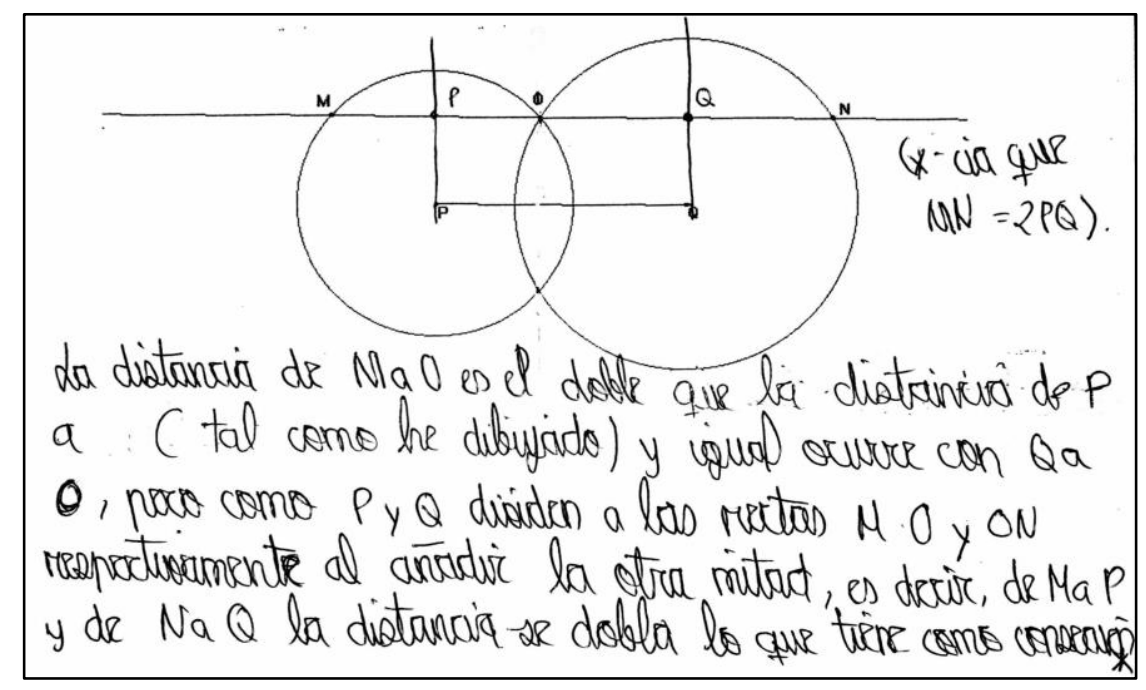

Figura 8 - Respuesta del alumno 14 a P4

Fuente: Datos de la investigación

A continuación, describimos el razonamiento configural que desarrolla el alumno para dar su respuesta a la tarea:

1) Aprehensión operativa de cambio figural: añade dos semirrectas al segmento PQ que parten de $\mathrm{P}$ y $\mathrm{Q}$ respectivamente.

2) Aprehensión operativa de identificación: identifica el segmento que forman los puntos MPO (este P, sobre el segmento MN, es distinto del punto $\mathrm{P}$ del enunciado).

3) Aprehensión discursiva: afirma que la distancia $\mathrm{M}$ a $\mathrm{O}$ es el doble que la distancia de $\mathrm{P}$ a O.

4) AOi: de manera análoga, identifica el segmento formado por los puntos OQN (este Q, sobre el segmento $\mathrm{MN}$, es distinto del punto Q del enunciado)

5) Aprehensión discursiva: igual ocurre con $Q$ a $O$. Es decir, afirma que la distancia de $\mathrm{O}$ a $\mathrm{N}$ es el doble de la distancia de $\mathrm{Q}$ a $\mathrm{O}$.

También, en este ejemplo se puede distinguir la realización del ciclo aprehensión discursiva/aprehensión operativa, si bien en este caso las asociaciones realizadas (por 
ejemplo, los dos segmentos MP y PO tienen longitudes iguales) no sean estrictamente con afirmaciones matemáticas. Aquí, la visión es el único proceso que guía la resolución del problema. Todo esto ocurre sin que se realice verificación alguna, más allá de la evidencia visual que proporciona la figura que acompaña al enunciado y las modificaciones que se han realizado sobre ella. El razonamiento configural realizado desemboca en lo que hemos denominado una conjetura sin demostración empírica.

$\mathrm{Su}$ respuesta traduce el razonamiento configural realizado al lenguaje natural. Si organizamos la estructura de su discurso, un tanto confuso, afirma que:

$\mathrm{MP} \equiv \mathrm{PO}$ y $\mathrm{OQ} \equiv \mathrm{QN}$ (Figura 9)

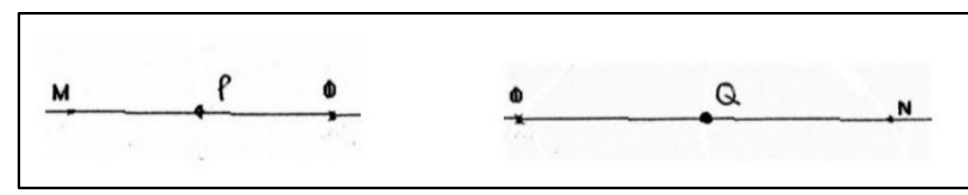

Figura 9 - Aprehensiones operativas de identificación Fuente: Datos de la investigación

y como PO + OQ = PQ es evidente visualmente (la respuesta del alumno refuerza esta idea denominando los puntos sobre la recta $\mathrm{MN}$ con las mismas letras que los centros de las circunferencias), entonces afirma que $\mathrm{MP}+\mathrm{QN}=\mathrm{PQ}$, por tanto, MN = $2 \mathrm{PQ}$ (Figura 10).

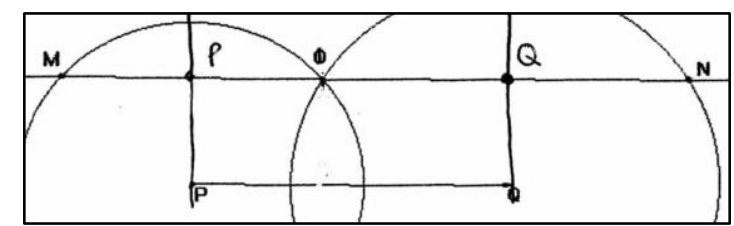

Figura 10 - Aprehensión de cambio figural en la respuesta del alumno 14 a P4 Fuente: Datos de la investigación

En el razonamiento configural propio de la explicación ninguna afirmación matemática (definición, teorema, ...) es asociada para la resolución del problema. Se produce una o varias aprehensiones operativas que convencen al alumno de la veracidad de la afirmación solicitada. Para ello, el estudiante verifica las afirmaciones que realiza mediante percepción. Este razonamiento configural es la solución del problema; la elaboración de un discurso para comunicar dicha solución es tan solo una conversión en el registro de representación semiótico, en este caso del registro figural al discursivo. Este discurso es una explicación que convence, pero no posee las características del discurso que denominamos demostración matemática.

Conjetura sin Demostración Conceptual $\leftrightarrow$ Prueba ingenua: se observa, en el razonamiento configural, la coordinación de aprehensiones discursivas y operativas necesarias en un contexto de Geometría Axiomática Natural para la demostración de una proposición. La respuesta tiene la organización discursiva propia de la demostración matemática, pero se 
acepta alguna o algunas afirmaciones matemáticas por procedimientos de validación perceptivos. La validez de todo el discurso descansa en la veracidad o no de las afirmaciones verificadas mediante procedimientos perceptivos, por lo que denominamos a las respuestas que produce este razonamiento configural Pruebas Ingenuas (Figura 11).

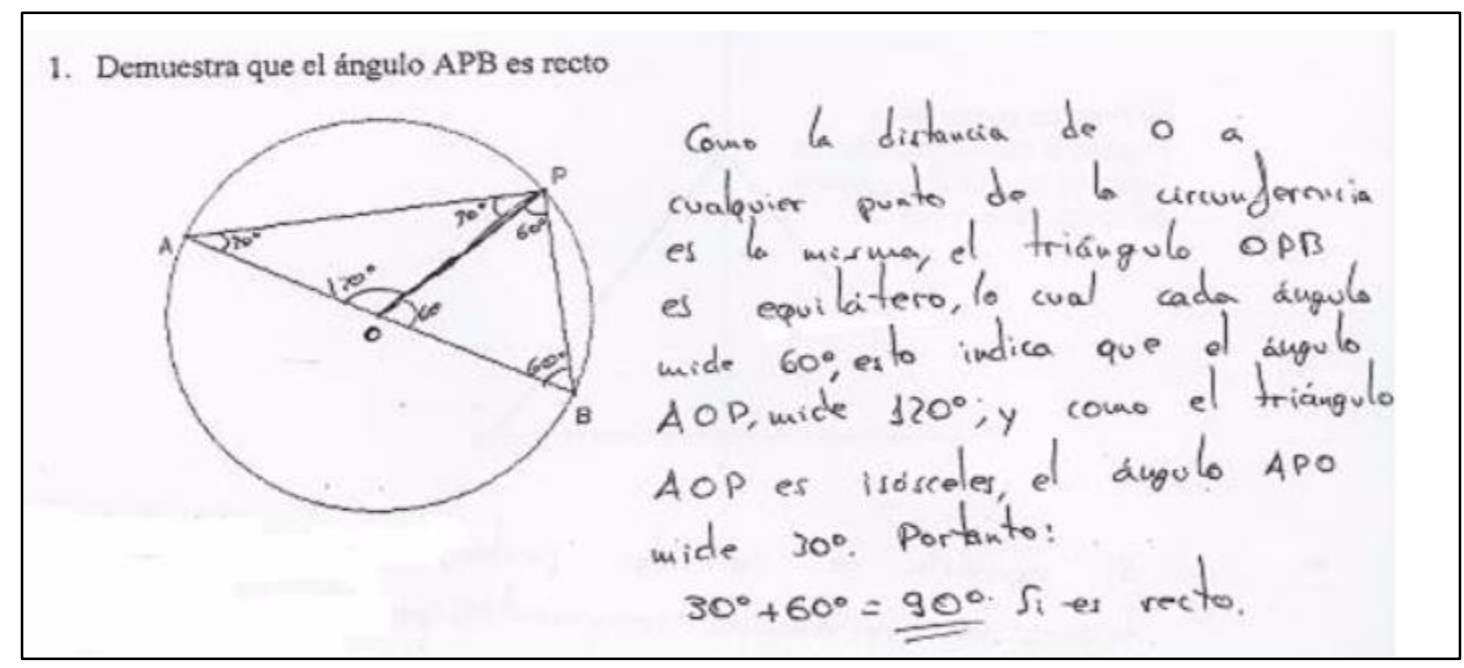

Figura 11 - Respuesta del alumno 32 a P1

Fuente: Datos de la investigación

Un problema de demostrar una propiedad geométrica en un contexto axiomático demanda una organización discursiva específica. A continuación, analizamos el razonamiento configural seguido por el estudiante en su respuesta.

1) Tras la aprehensión operativa de identificación, el estudiante realiza una aprehensión visual-discursiva (aprehensión discursiva con cambio de anclaje de visual a discursivo - Figura 12)

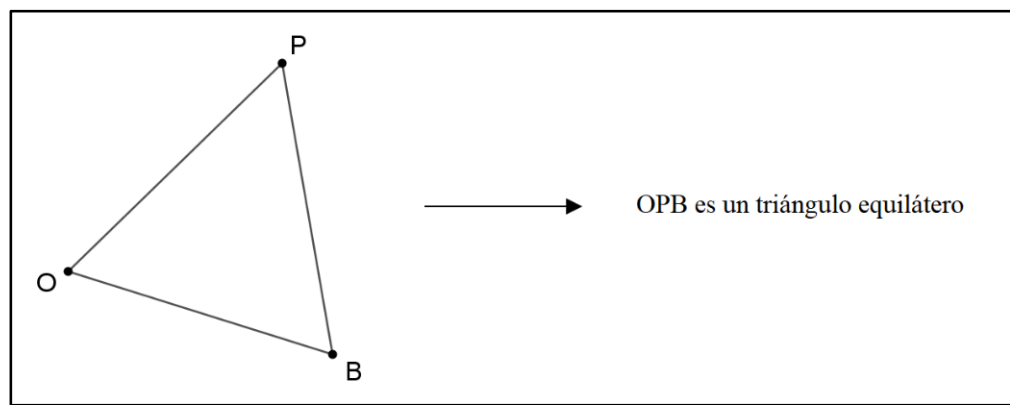

Figura 12 - Aprehensión visual-discursiva

Fuente: Datos de la investigación

2) Realiza la siguiente aprehensión operativa de identificación para asociar la siguiente afirmación matemática (aprehensión visual-discursiva - Figura 13). 


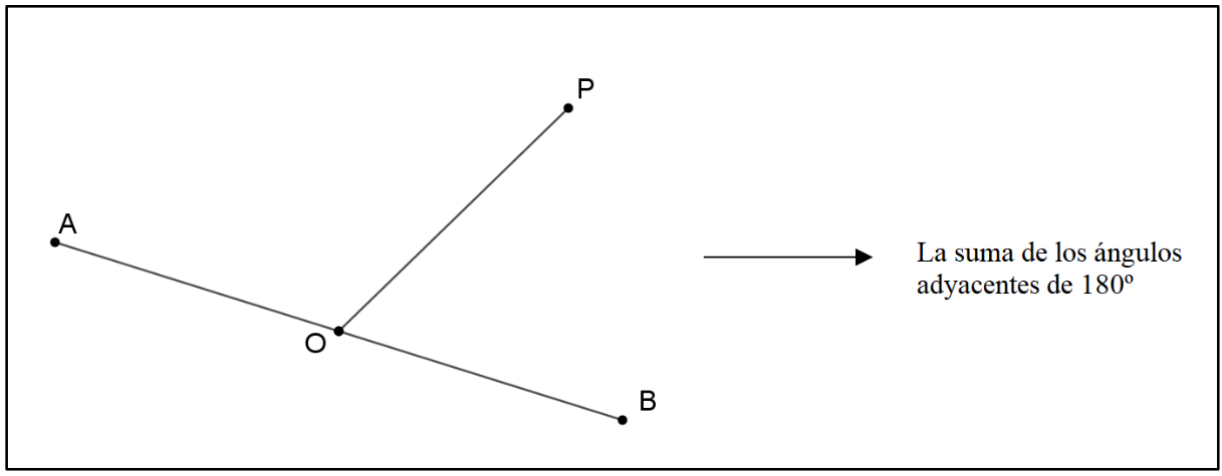

Figura 13 - Aprehensión visual-discursiva Fuente: Datos de la investigación

3) Por último, realiza la siguiente aprehensión operativa de identificación (Figura 14).

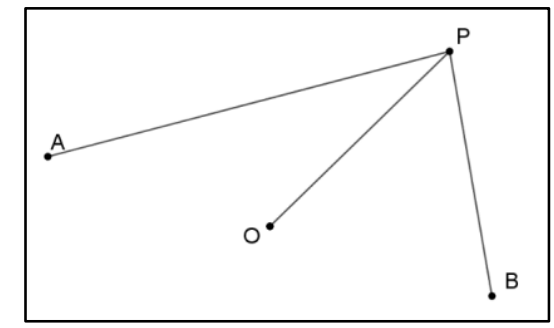

Figura 14 - Aprehensión operativa de identificación Fuente: Datos de la investigación

$\mathrm{Su}$ razonamiento configural desemboca en lo que hemos denominado una Conjetura sin Demostración Conceptual, que le permite organizar un discurso que analizamos a continuación, en la Figura 15.

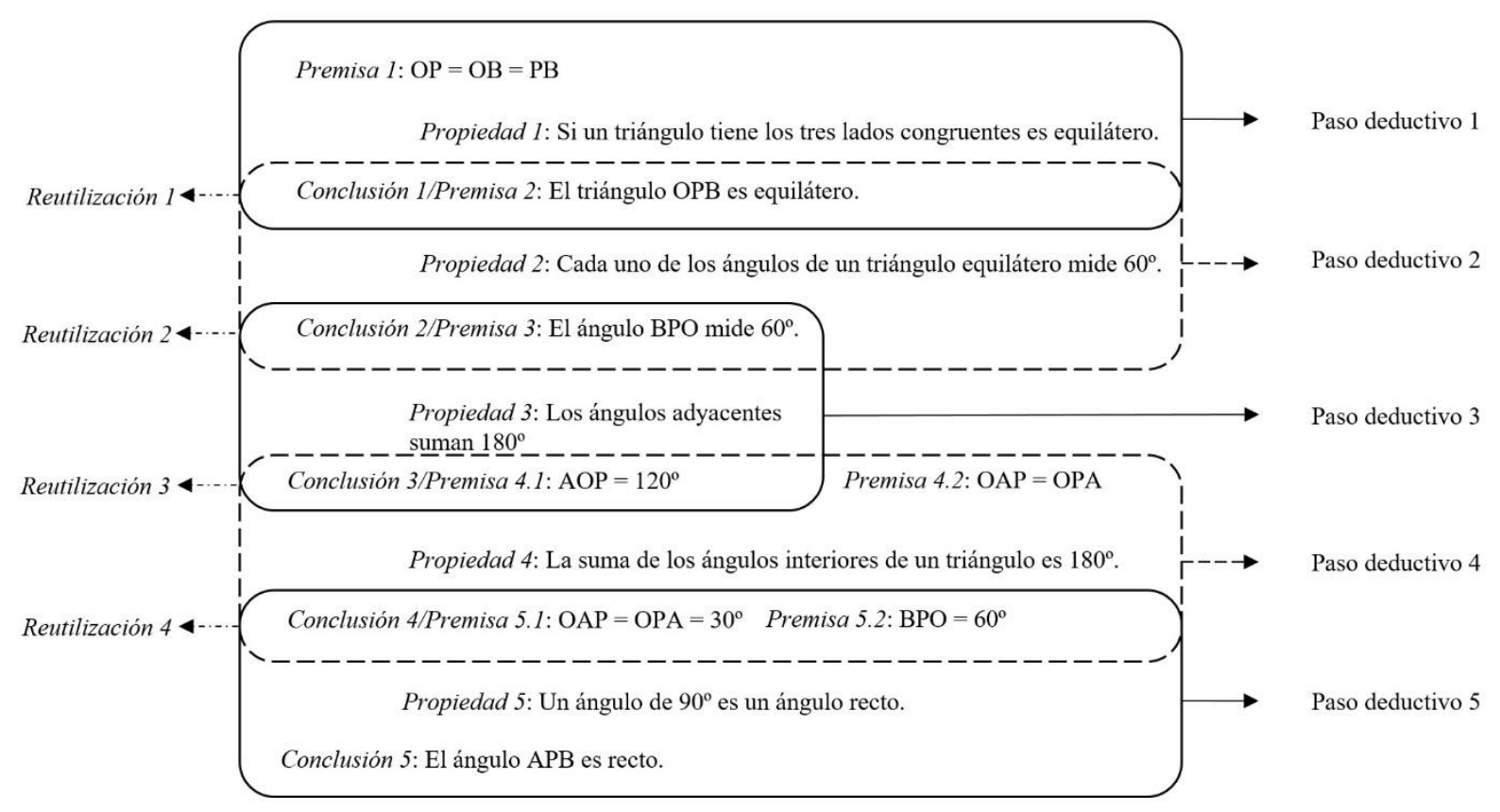

Figura 15 - Organización discursiva de la respuesta del alumno 32 a P1 Fuente: Datos de la investigación 
Del análisis conjunto de su razonamiento configural y de su organización discursiva podemos inferir que, a pesar de un discurso poco elaborado, el estudiante conoce la organización específica de la demostración matemática ya que realiza pasos deductivos en los que el estatus de cada proposición (premisa, propiedad o conclusión) es el correcto. No obstante, comete un error al principio de su razonamiento: utiliza una premisa falsa $(\mathrm{OB} \equiv \mathrm{PB})$, verdadera para él porque resulta visualmente aceptable en la imagen que acompaña al enunciado (entrevista). Este es uno de los errores más comunes de los estudiantes que transitan desde de la Geometría Natural a la Geometría Natural Axiomática: la validación de alguna de las premisas mediante procedimientos de verificación perceptivos sin uso de instrumentos (PRIOR; TORREGROSA, 2013).

Como vimos anteriormente, la validación de una proposición mediante procedimientos perceptivos es un recurso natural y común en la resolución de problemas geométricos empíricos dentro del contexto escolar. El uso de este recurso en el contexto demostrativo se suma a la teoría de que no existe un paso natural desde la argumentación a la demostración matemática. Se hace necesaria una ruptura con la práctica matemática escolar y una enseñanza específica de las características de la demostración matemática.

Truncamiento $\leftrightarrow$ Demostración: la respuesta al problema 3 del alumno 26 que mostramos en el apartado de análisis es un ejemplo del binomio Truncamiento Demostración que describimos en este apartado.

$\mathrm{Su}$ razonamiento configural desemboca en un truncamiento, único desenlace posible cuando se alcanza la solución al problema en el contexto de la Geometría Axiomática Natural. La validez de sus afirmaciones depende, siempre, de su estatus operativo, y nunca de su contenido, por lo que ninguna afirmación es validada mediante procedimientos perceptivos. Aunque el razonamiento configural guía el discurso generado, proporcionando las subconfiguraciones y las afirmaciones matemáticas pertinentes para la solución del problema, el discurso (demostración), una vez generado, es completamente independiente, pues la estructura del paso deductivo y la concatenación de pasos por solapamiento es una operación puramente discursiva. Es decir, la demostración es una operación discursiva que tiene entidad independientemente del razonamiento configural que haya guiado su construcción; su validez descansa únicamente en las operaciones proposicionales (paso deductivo y solapamiento de proposiciones) que conectan las hipótesis del enunciado con la tesis solicitada.

El estudio de las respuestas de alumnos de 15-16 años a un cuestionario proporcionado por el investigador nos ha permitido refinar nuestro modelo sobre el razonamiento configural, añadiendo a los desenlaces descritos anteriormente (Truncamiento, Conjetura sin 
Demostración Empírica y Conjetura sin Demostración Conceptual), un nuevo desenlace que denominamos Truncamiento Naif, que describe el razonamiento configural necesario para la comprobación de un caso particular en el espacio de trabajo que hemos denominado Geometría Natural.

\section{Discusión y conclusiones}

El análisis del razonamiento configural utilizado y de la organización discursiva de las producciones de estudiantes de secundaria y universidad, cuando resuelven problemas geométricos de probar, nos ha llevado a plantear el siguiente esquema en el que se describe el razonamiento configural propio de cada uno de los espacios de trabajo geométrico; así como estadios de transición desde la Geometría Natural hasta la Geometría Axiomática Natural (Figura 16).

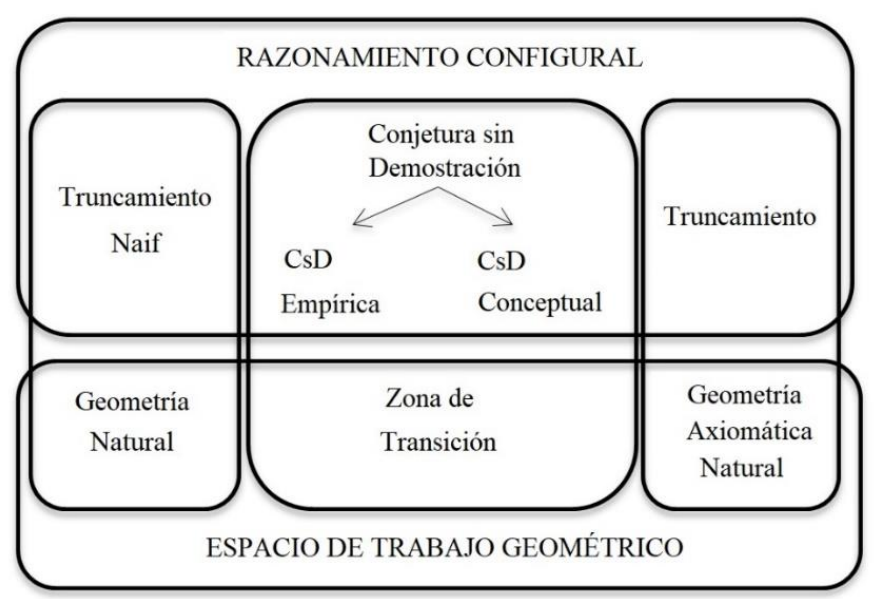

Figura 16 - Razonamiento configural y espacio de trabajo geométrico Fuente: Datos de la investigación

Nuestro modelo de razonamiento configural se muestra como un instrumento con gran poder explicativo de las interacciones que se producen entre los distintos procesos involucrados en la actividad geométrica. La comprensión de su funcionamiento y de los factores que inciden en sus distintos desenlaces nos ayuda a entender y a explicar la variedad de comportamientos que observamos en los alumnos cuando resuelven problemas geométricos.

El estudio conjunto del razonamiento configural y de las organizaciones discursivas de las respuestas de los alumnos nos ha permitido desarrollar un modelo teórico que describimos en la Figura 16. Éste nos proporciona un marco teórico para analizar las respuestas de los estudiantes que resuelven problemas geométricos: nos informa de las creencias del alumno 
respecto al contexto en el que tiene lugar la actividad matemática (espacio de trabajo geométrico), de su conciencia sobre el alcance de una proposición matemática (caso general o particular), de su conocimiento sobre lo que una organización discursiva produce (explicación o prueba matemática), así como de su bagaje de conocimientos matemáticos (aprehensiones discursivas), de sus capacidades de visualización (aprehensiones operativas), y de la coordinación de éstas (razonamiento configural) para la resolución de problemas geométricos.

El estudio también ha puesto de manifiesto los dos obstáculos epistemológicos que dificultan el tránsito desde la Geometría Natural, propia de las etapas elementales, hasta la Geometría Axiomática Natural, que debe desarrollarse al final de la etapa secundaria y que sienta las bases para el acceso a la Geometría Formal Axiomática. Estos son: la distinción entre dibujo (una representación particular de una figura) y figura (objeto geométrico abstracto caracterizado por las propiedades matemáticas que lo definen, considerada en HERSHKOWITZ; PARZYSZ; VAN DORMOLEN, 1996); la comprensión de lo que una prueba matemática produce (cambio en el valor lógico de la proposición dentro del marco teórico en el que es enunciada, analizada en DUVAL, 2007).

Consideramos que análisis posteriores de este último obstáculo, relativo al origen del valor de las distintas proposiciones que se organizan en un discurso, pueden proporcionar una mejor comprensión de los diferentes estadios por los que un estudiante debe transitar desde las primeras justificaciones elementales hasta alcanzar una necesaria comprensión de la naturaleza de la prueba matemática.

Una cuestión interesante para abordar en nuevas investigaciones podría ser: ¿la respuesta fuera del ETG en el que se plantea la tarea es producto de una incomprensión o fruto de la necesidad de dar una respuesta? Es decir, el alumno que ofrece una respuesta en un ETG que no coincide con el ETG idóneo, ¿es consciente de su validez o no?

\section{Referencias}

BALACHEFF, N. Une étude des processus de preuve en mathématiques chez des élèves de Collège. Thèse (Doctorat en sciences didactiques mathématiques) - Université Joseph Fourier, Grenoble, 1988.

CLEMENTE, F.; LLINARES, S. Formas de discurso y razonamiento configural de estudiantes para maestro en la resolución de problemas de geometría. Enseñanza de las Ciencias, Barcelona, v. 33, n. 1, p. 9-27, 2015.

CLEMENTE, F.; TORREGROSA, G.; LLINARES, S. La identificación de figuras prototípicas en el desarrollo del razonamiento configural. In: SCOTT, P.; RUÍZ, A. (Ed.). CONFERENCIA INTERAMERICANA DE EDUCACIÓN MATEMÁTICA (CIAEM-IACME), 14, 2015, Tuxtla 
Gutiérrez. Educación Matemática en las Américas: 2015. Tuxtla Gutiérrez: Comité Interamericano de Educación Matemática, 2015. v. 9, p.130-140.

CLEMENTE, F.; TORREGROSA, G.; LLINARES, S. Visualization and Configural Reasoning. Bolema, Rio Claro, v. 31, n. 57, p. 497-516, 2017.

DUVAL, R. Geometry from a cognitive point of view. In: MAMMANA, C.; VILLANI, V. (Ed.). Perspective on the Teaching of Geometry for the 21st Century. Dordrecht/Boston: Kluver Academic Publishers, 1998. p. 37-51.

DUVAL, R. Un tema crucial en la educación matemática: La habilidad para cambiar el registro de representación. La Gaceta de la Real Sociedad Matemática Española, Madrid, v. 9, n. 1, p. $143-$ 168, 2006.

DUVAL, R. Cognitive functioning and the understanding of mathematical processes of proof. In: BOERO, P. (Ed.). Theorems in schools: from history and cognition to classroom practice. Rotterdam: Sense Publishers, 2007. p. 137-161.

FIALLO, J.; CAMARGO, L.; GUTIÉRREZ, A. Acerca de la enseñanza y el aprendizaje de la demostración en matemáticas. Revista Integración, Bucaramanga, v. 31, n. 2, p. 181-205, 2013.

HAREL, G.; SOWDER, L. Student's Proof Schemes: Results from exploratory studies. In DUBINSKY, E.; SCHOENDFELD, A.; KAPUT, J. (Ed.). Research on Collegiate Mathematics Education. Providence: American Mathematical Society, 1998. p. 234-283.

HERSHKOWITZ, R.; PARZYSZ, B.; VAN DORMOLEN, J. Space and Shape. In: BISHOP, A.J.; CLEMENTS, M.A.; KEITEL, C.; KILPATRICK, J.; LABORDE, C. (Ed.). International handbook of Mathematics Education. Dordrecht: Kluver Academic Publishers, 1996. p. 161-204.

HOUDEMENT, C.; KUZNIAK, A. Un exemple de cadre conceptuel pour l'étude de l'enseignement de la géométrie en formation des maîtres. Educational Studies in Mathematics, Berlín, v. 40, p. 283 312, 1999.

HOUDEMENT, C.; KUZNIAK, A. Paradigmes géométriques et enseignement de la géométrie. Annales de didactique et de sciences cognitives, Estrasburgo, v. 11, p. 175-193, 2006.

KUZNIAK, A. Personal Geometrical Working Space: A Didactic and Statistical Approach. Studies in Computational Intelligence (SCI), Berlín, v. 127, p.185-202, 2008.

LARIOS, V. La construcción de la prueba geométrica en un ambiente de geometría dinámica en secundaria. In: LEZAMA, J.; SÁNCHEZ, M.; MOLINA, J. (Ed.). Acta Latinoamericana de Matemática Educativa A.C. Coalcaco, 2005. p. 765-770.

LLINARES, S.; CLEMENTE, F. Characteristics of pre-service primary school teachers' configural reasoning. Mathematical Thinking and Learning, Londres, v. 16, n. 3, p. 234-250, 2014.

MARIOTTI, M.A.; PEDEMONTE, B. Intuition and proof in the solution of conjecturing problems'. ZDM, Berlín, v. 51, p. 759-777, 2019.

MARRADES, R.; GUTIÉRREZ, Á. Proofs produced by secondary school students learning geometry in a dynamic computer environment. Educational Studies in Mathematics, Berlín, v. 44, p. 87-125, 2000 .

PARZYSZ, B. Knowing vs Seeing. Problems of the plane representation of space geometry figures. Educational Studies in Mathematics, Berlín, v.19, n. 1, p. 79-92, 1988. 
PARASKEVI, M.; GAGATSIS, A. Ambiguity in the way of looking at geometrical figures. Revista Latinoamericana de Investigación en Matemática Educativa, Ciudad de México, v. 17, n. 4, p. $165-179,2014$

PRIOR, J.; TORREGROSA, G. Razonamiento configural y procedimientos de verificación en contexto geométrico. Revista Latinoamericana de Investigación en Matemática Educativa, Ciudad de México, v. 16, n. 3, p. 339-368, 2013.

SAORÍN, A.; TORREGROSA, G.; QUESADA, H. Razonamiento configural extendido: coordinación de procesos cognitivos en la resolución de problemas empíricos. In: CONGRESO DE EDUCACIÓN MATEMÁTICA DE AMÉRICA CENTRAL Y EL CARIBE (CEMACYC), 2, 2017, Cali. Memorias del II Congreso de Educación Matemática de América Central y El Caribe. México: Comité Interamericano de Educación Matemática, 2019. p. 102-110.

STYLIANIDES, G.; STYLIANIDES, A. Facilitating the transition from empirical arguments to proof. Journal for Research in Mathematics Education, Reston, v. 40, n. 3, p. 314-352, 2009.

TORREGROSA, G.; QUESADA, H. Coordinación de procesos cognitivos en geometría. Revista Latinoamericana de investigación en Matemática Educativa, Ciudad de México, v. 10, n. 2, p. 275-300, 2007.

TORREGROSA, G.; QUESADA, H.; PENALVA, C. Razonamiento configural como coordinación de procesos de visualización. Enseñanza de las Ciencias, Barcelona, v. 28, n. 3, p. 327-340, 2010.

TORREGROSA, G. Coordinación de procesos cognitivos en la resolución de problemas: relación entre geometría y álgebra. Avances en Investigación en Educación Matemática, Badajoz, v. 12, p. $1-17,2017$.

ZAZKIS, D.; WEBER, K.; MEJÍA-RAMOS, J. Bridging the gap between graphical arguments and verbal-symbolic proofs in a real analysis context. Educational Studies in Mathematics, Berlín, v. 93, p.155-173, 2016.

Submetido em 03 de Março de 2019 Aprovado em 09 de Setembro de 2019. 


\section{ERRATA}

No artigo "Razonamiento Configural y Espacio de Trabajo Geométrico en la Resolución de Problemas de Probar", com número de DOI: http://dx.doi.org/10.1590/1980-4415v34n66a09, publicado no periódico Bolema, 34 (66): 178-198, na página 178:

Se faz necessário indicar que os sobrenomes dos autores a serem referenciados devem ser aqueles destacados em negrito:

Juan Prior Martínez

Germán Torregrosa Gironés 